\title{
The RANKL/RANK system in female reproductive organ tumors: A preclinical and clinical overview
}

\author{
Ana Monzó-Miralles ${ }^{1,2, A, E, F}$, Víctor Martín-González ${ }^{3, A, E, F}$, Sara Smith-Ballester ${ }^{4, A, E, F}$, \\ Victoria Iglesias-Miguel ${ }^{4, A, E, F}$, Antonio Cano $0^{4, A, D-F}$ \\ 1 Service of Obstetrics and Gynecology "La Fe", Hospital Universitari i Politècnic La Fe, Valencia, Spain \\ ${ }^{2}$ Department of Pediatrics, Obstetrics and Gynecology, University of Valencia, Spain \\ ${ }^{3}$ Service of Obstetrics and Gynecology, Hospital Clínico Universitario-INCLIVA, Valencia, Spain \\ ${ }^{4}$ University of Valencia and Service of Obstetrics and Gynecology, Hospital Clínico Universitario-INCLIVA, Spain \\ A - research concept and design; B - collection and/or assembly of data; $\mathrm{C}$ - data analysis and interpretation; \\ $D$ - writing the article; $E$ - critical revision of the article; $F$ - final approval of the article
}

\section{Address for correspondence}

Antonio Cano

E-mail: Antonio.cano@uv.es

\section{Funding sources}

This research received funding from the Project EURODIET, jointly funded by the action Impact of Diet, Food Components and Food Processing on Body Weight Regulation and Overweight Related Metabolic Diseases (METADIS 2019) of the European Union's H2020 Research and Innovation Program (grant No. 696300), and Health Institute Carlos III, Spain (Project AC19/00084), with the support of the European Regional Development Fund (ERDF).

Conflict of interest

None declared

Received on July 16, 2021

Accepted on July 21, 2021

Published online on August 24, 2021

Cite as

Monzó-Miralles A, Martín-González V, Smith-Ballester S, Iglesias-Miguel V, Cano A. The RANKL/RANK system in female reproductive organ tumors: $A$ preclinical and clinical overview. Adv Clin Exp Med. 2021;30(9):879-883. doi:10.17219/acem/140422

DOI

10.17219/acem/140422

Copyright

Copyright by Author(s)

This is an article distributed under the terms of the

Creative Commons Attribution 3.0 Unported (CC BY 3.0)

(https://creativecommons.org/licenses/by/3.0/)

\begin{abstract}
The receptor activator of nuclear factor-KB (RANK) and its ligand RANKL are members of the tumor necrosis factor (TNF) super-family of cytokines with a role in progestogen-associated malignancies in breast. Basic and clinical data support the participation of the cytokine pathway in metastatic disease and as poor prognosis markers. The value of RANK/RANKL as prognostic indicators in endometrial and ovarian tumors, as well as the data suggesting a potential role of RANK/RANKL in hormone dependent tumorigenesis in the endometrium, have been described. The D-CARE study could not confirm benefit in the modulation of RANKL in breast cancer.
\end{abstract}

Key words: RANK, RANKL, cancer, breast, genital tract

\section{Background}

Cancers of the reproductive tissues have a particularly predominant place in the global burden of cancer in women. According to the American Cancer Society, the 6 most frequently diagnosed tumors in women are breast cancer (30\%), lung and bronchus (13\%), colon and rectum (8\%), uterine corpus (7\%), skin melanoma (5\%), and non-Hodgkin lymphoma (4\%). ${ }^{1}$ The picture changes for mortality, in which lung and bronchus ranks $1^{\text {st }}(22 \%)$, breast $2^{\text {nd }}(15 \%)$, followed by colon and rectum (8\%), pancreas (8\%), ovary (5\%), and uterine corpus (4\%). Accordingly, 3 reproductive organ-related cancers, breast, uterus and ovary, are among the 6 most prevalent and deadliest tumors in women.

There is a well-established role for ovarian steroids in the genesis of tumors affecting the female reproductive organs. Associations have been shown not only in epidemiological data, but also via clear biological mechanisms. For example, the proliferative role of estrogens in the endometrial epithelium has been confirmed under different experimental conditions, including cell lines and animal models, and in humans. 


\section{Molecular background}

\section{Estrogen and the endometrium}

Estrogen receptors (ER) are highly expressed in both epithelium and stroma in the human endometrium, and ER activation leads to a proliferative effect, as shown throughout the physiological menstrual cycle and in classic studies in the human endometrium. ${ }^{2,3}$

Considerable advances have also been made by studying the molecular mechanisms underlying these observations. ${ }^{4}$ Indeed, the ER transcription apparatus has been linked to the activation of a long list of genes that control multiple cellular pathways. These include mitogenic actions matching those of human cancer, including rapidly increased $c-M y c$ and $c y c l i n D 1$ expression. Angiogenesis, a crucial mechanism linked to tumor progression, is also promoted by estrogen; although its action, if any, is limited to inducing metastasis. ${ }^{4}$ Consistent with these actions, estrogen-only use has been associated with an increased risk of endometrial cancer in clinical studies of menopausal women. ${ }^{5}$ The association is less clear in the breast; ${ }^{6}$ although, limiting estrogen exposure with aromatase inhibitors ${ }^{7}$ or ER blockade with selective estrogen receptor modulators $(\mathrm{SERMs})^{8}$ have been shown to reduce breast cancer risk.

Early work confirmed that progesterone receptors (PR) are a product of ER gene activation, ${ }^{9}$ which was followed by finding that PR activation in the endometrium limited the mitogenic effect of estrogens. ${ }^{3}$ This anti-proliferative action of progestogens is the reason behind recommending progestogen use in combination with estrogen in women who have not undergone hysterectomy. ${ }^{10}$ There is consistent clinical evidence showing that adding progestogens to estrogens in hormonal therapy reduces endometrial cancer risk to similar rates as in untreated women. ${ }^{5}$

\section{Progestogens and the breast}

A series of findings prompted the hypothesis that the anti-proliferative action of progestogens observed in the endometrium might not be replicated in the breast. Proliferation was increased in breast epithelium during the luteal phase, a finding that contrasted with its quiescent status in the endometrium. ${ }^{11}$ Observational studies and clinical trials subsequently confirmed that progestogens and estrogens combined result in a higher risk than is associated with estrogen-only treatment. ${ }^{6}$ These findings have also been observed for progestogen-only treatments in hormonal contraceptive users. ${ }^{12}$ The molecular mechanism underlying the proliferative effect of progestogens seen in breast but not endometrium has remained elusive for several years.

\section{RANK/RANKL and hormone-linked tumorigenesis}

Studies in recent years have contributed pivotal data on the oncogenic role of progestogens and the involvement of the receptor activator of nuclear factor- $\mathrm{kB}$ (RANK) and its ligand, RANKL. RANK and RANKL are members of the tumor necrosis factor (TNF) super-family of cytokines. RANK was identified in 1997 in the T cell membrane, where it showed a modulating role in the interaction of T cells and dendritic cells. ${ }^{13}$ RANK/RANKL have emerged as key elements in various immune response processes, as well as in mechanisms linked to inflammation, organogenesis and apoptosis. ${ }^{14,15}$ A fundamental role has also been found in bone metabolism regulation, in which they act mainly by promoting the differentiation and activation of osteoclast progenitors. ${ }^{16}$

The RANKL ligand participates in the growth and differentiation of the epithelial component in mammary ducts and lobes during puberty and the phases of the menstrual cycle $^{17}$, as shown in both animal and human research. Likewise, RANK/RANKL have been proposed as key mediators in the proliferative action of progestogens in the breast. ${ }^{18,19}$

\section{RANK/RANKL and breast tumorigenesis as a model}

The current understanding concerning the role of RANK/RANKL in tumorigenesis stems largely from studies in the breast. Mapping studies showed that RANKL expression increases during gestation, expressed mainly in the ductal luminal area and in developing alveoli, while RANK is expressed in the basal layers of the ductal epithelium. ${ }^{17}$ Experiments in transgenic animals clearly showed the role of RANK/RANKL in proliferative and anti-apoptotic mechanisms. ${ }^{20}$

RANK/RANKL involvement in hormonal tumorigenesis is suggested by various findings: i) ER and PR are coexpressed with RANKL in luminal epithelium; ii) there is a confirmed correlation between RANKL expression and circulating levels of progesterone in humans, in both normal and malignant cells ${ }^{21,22}$; iii) the induction of mammary tumors by progestogens is accompanied by a striking overexpression of RANKL in PR-positive luminal cells ${ }^{19,23}$; and iv) RANK blockade is associated with tumor differentiation and reduced propensity for recurrence. ${ }^{24}$

\section{Molecular mechanisms of RANKL/ RANK in breast tumorigenesis}

Animal models have revealed a perfectly coordinated apparatus involving progesterone-induced proliferation and synthesis of RANKL in luminal cells expressing PR, and paracrine-induced proliferation by RANKL in neighboring cells lacking PR (Fig. 1). In the presence of susceptibility 


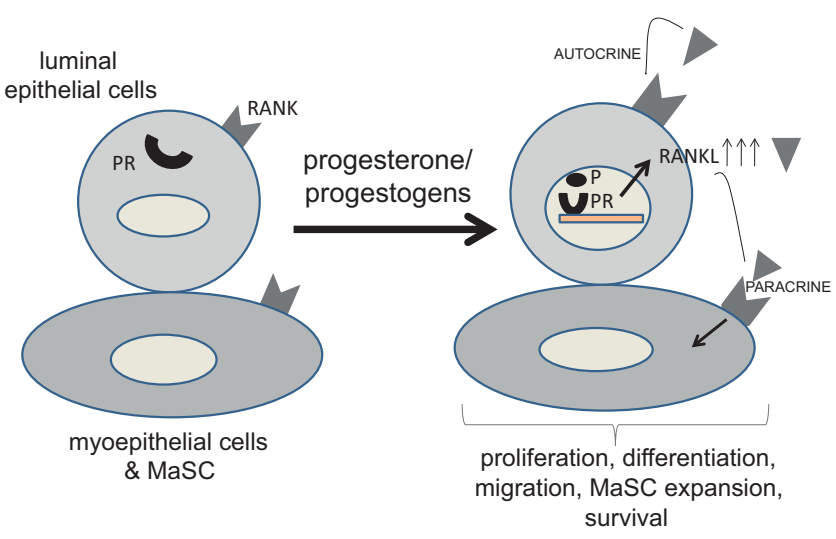

Fig. 1. The image shows RANK expressed in luminal, myoepithelial, and mammary stem cells (MaSC). Luminal cells also express progesterone receptors (PR), which upon stimulation induce RANKL synthesis. In an autocrine or paracrine form, RANKL activates RANK to promote proliferation, migration, MaSC expansion and cell survival ${ }^{18}$

agents like carcinogens or genetic lesions, persistent proliferative stimuli may lead to hyperplasia and neoplastic transformation.

Data from human studies have reinforced findings from preclinical studies. For example, malignant mammary cells express RANK and RANKL, ${ }^{23}$ and more intriguingly, RANK expression has been correlated with tumor aggressiveness in women. ${ }^{25} \mathrm{~A}$ crucial notion in this regard is whether RANK/RANKL overexpression following malignancy onset feeds cellular machinery involved in dedifferentiation and aggressive tumor behavior, or rather represents a mere epiphenomena associated with the malignant phenotype.

\section{RANK/RANKL and tumor aggressiveness}

Beyond data confirming a role for RANK/RANKL in channeling the oncogenic role of progestogens, other findings suggest that these cytokines could constitute preferential pathways to cancer, independently of their relationship with these steroids.

Along these lines, interesting results were derived from studies in carriers of the susceptibility genes $B R C A 1$ and $B R C A 2$, which confer increased risk for more aggressive mammary tumor patterns, often ER- and PR-negative tumors. A multicenter study compared circulating levels of osteoprotegerin (OPG), which exhibits affinity and acts as a blocking agent of RANKL, between $B R C A 1 / 2$ carriers $(\mathrm{n}=391)$ and non-carriers $(\mathrm{n}=782) . B R C A 1 / 2$ carriers had lower serum free levels of OPG, implying that RANKL activity was probably higher. ${ }^{26}$ Of particular interest, the levels of OPG were inversely correlated with the detection of germ mutations known to confer a higher risk for breast cancer in BRCA carriers. ${ }^{26}$

Other research has found that the RANK/RANKL system plays a role in malignant drift in cancers in $B R C A$ $1 / 2$ carriers, in both experimental models ${ }^{27}$ and clinical studies. ${ }^{28}$ These findings have fueled interest in a proposal of RANKL blockade with anti-RANKL antibodies, such as denosumab, as a possible way to reduce cancer risk in $B R C A 1$ carriers. ${ }^{29}$

Another key feature ascribed to RANK/RANKL is their value as indicators of poor tumor prognosis. In this regard, increased RANK expression has been found in hormone receptor-negative mammary tumors. ${ }^{30}$ There are also some indications of metastatic potential, another feature related to tumor aggressiveness. Treatment with OPG reduced bone metastases in a mouse model of melanoma metastasis; although, the effect seemed limited to bone, without extending to other organs. ${ }^{31}$

\section{Endometrium as a new scenario}

The human menstrual cycle clearly reflects the opposing actions of estrogen and progesterone on the proliferative response of endometrial epithelium. A rapid proliferative response during the follicular phase is soon downregulated by progesterone, which is synthesized by the corpus luteum after ovulation. Progesterone therefore has an anti-proliferative effect in endometrium, in contrast to reported findings in the breast. While the molecular details underlying these opposing actions are not well understood, the question has arisen as to whether RANKL/RANK participate in endometrial proliferative events.

A first step has been taken to investigate whether RANK/ RANKL are expressed in human endometrium. Recent work identified RANK in human endometrial sections by immunohistochemistry (Fig. 2). ${ }^{32}$ Moreover, estrogens are associated with an increased risk of type I endometrial tumors, and also type II tumors, albeit to a lesser extent. ${ }^{33}$ However, and against what has been observed in the breast, it is unclear in the endometrium whether RANKL/RANK is involved in the process of estrogenmediated tumorigenesis.

More data have been published concerning the roles of RANK/RANKL as prognostic indicators in endometrial cancer. Analysis of data obtained by immunohistochemistry in a series of tumors has shown that, as for the breast, RANK expression is associated with indicators of poor prognosis, such as grade of differentiation or tumor stage, ${ }^{32}$ and also with myometrial invasion, lymph node metastasis, and lymphovascular space involvement. ${ }^{34}$ Remarkably, RANK activation with RANKL reproduced findings similar to the aforementioned cell proliferation; although, in this case, in an endometrial tumor cell line. This effect was downregulated by medroxyprogesterone acetate (MPA), a progestogen repeatedly shown to limit the progression of PR-positive endometrial cancers.

The conclusion drawn from these experiments is that, in endometrial cancer cell lines, RANKL reproduced the proliferative and anti-apoptotic effect observed in the breast. However, and against the observations 


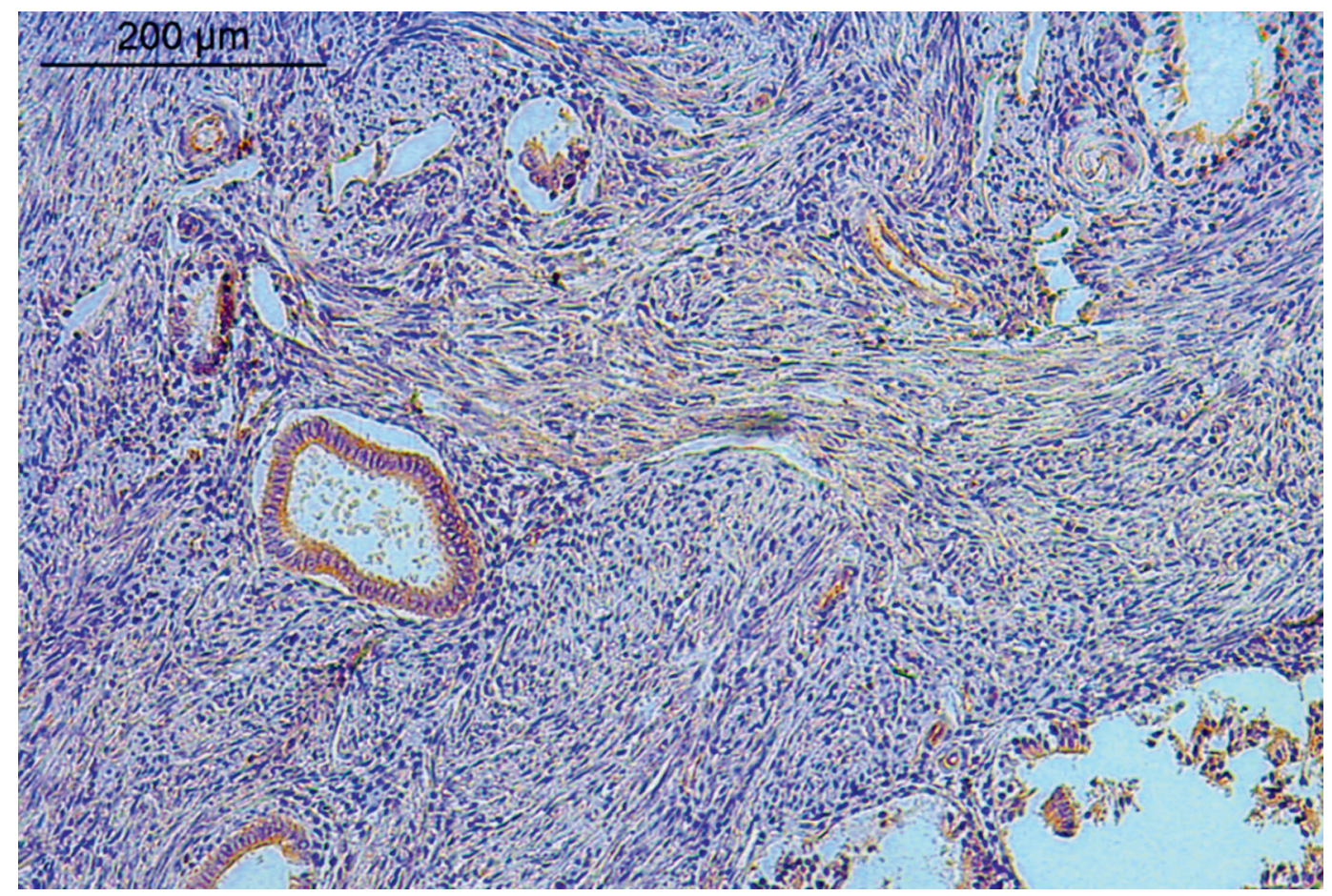

Fig. 2. The receptor activator of nuclear factor-KB (RANK) immunohistochemical staining (brown) in normal endometrium. Faint positive staining was found across luminal and glandular epithelium and stroma. The density of RANK staining increases in malignant endometrial tumors (not shown) and the signal increases with tumor de-differentiation; $\times 100$ magnification ${ }^{32}$

in the breast, the progestogen (MPA) limited the expansive effects mediated by RANKL in the endometrial tumor. The molecular basis for the opposite action of progestogens in breast and endometrium is unknown.

\section{Ovarian cancer}

Studies have been published linking hormonal therapy use with risk of ovarian cancer, ${ }^{35}$ yet the potential involvement of RANKL/RANK in ovarian tumorigenesis is still unclear, and data are still very sparse. Interestingly, as shown in endometrium and breast, increased RANKL expression has been found in ovarian cancer, where higher expression was associated with poor prognosis indicators, specifically with reduced progression-free and overall survival. ${ }^{36}$

\section{Conclusions}

A wealth of data has accumulated over recent years implicating RANKL/RANK in both tumorigenesis and prognosis in several reproductive system cancers, including breast and endometrium. The only confirmed role in both endometrial and ovarian cancer is that of prognostic indicator, which may involve either RANK or RANKL depending on the study. Experiments with endometrial cancer cell lines have shown that, in parallel with findings in breast cancer, RANK/RANKL are involved in proliferation and migration mechanisms.

These data have aroused interest in the potentially beneficial effect of blocking RANKL to improve prognosis in women with any of the cancer types studied. Unfortunately, available studies are inconclusive. The D-CARE study randomized 4509 women with stage II or III breast cancer to denosumab or placebo. The primary endpoint was bone metastasis-free survival, for which no betweengroup differences were found. ${ }^{37}$ However, time to first skeletal-related effect and time to bone metastasis progression were significantly lower in women with metastatic breast cancer, receiving denosumab when they expressed RANK on circulating tumor cells. ${ }^{38}$ Consequently, further research is needed to better define the role of RANK/RANKL in tumors of the reproductive organs. Only through enhanced understanding will appropriate strategies utilizing modulators such as denosumab be designed.

\section{ORCID iDs}

Ana Monzó-Miralles (1) https://orcid.org/0000-0003-4509-0065 Víctor Martín-González (1) https://orcid.org/0000-0002-2989-4883 Sara Smith-Ballester (1) https://orcid.org/0000-0003-4117-7454 Victoria Iglesias-Miguel (1) https://orcid.org/0000-0002-9334-3920 Antonio Cano (1) https://orcid.org/0000-0001-8046-0303

\section{References}

1. Siegel RL, Miller KD, Fuchs HE, Jemal A. Cancer statistics, 2021. CA Cancer J Clin. 2021;71(1):7-33. doi:10.3322/caac.21654

2. Lessey BA, Killam AP, Metzger DA, Haney AF, Greene GL, McCarty KS Jr. Immunohistochemical analysis of human uterine estrogen and progesterone receptors throughout the menstrual cycle.J Clin Endocrinol Metab. 1988;67(2):334-340. doi:10.1210/jcem-67-2-334

3. Whitehead MI, Townsend PT, Pryse-Davies J, Ryder TA, King RJ. Effects of estrogens and progestins on the biochemistry and morphology of the postmenopausal endometrium. N Engl J Med. 1981; 305(27):1599-1605. doi:10.1056/NEJM198112313052701

4. Liang J, Shang Y. Estrogen and cancer. Annu Rev Physiol. 2013;75: 225-240. doi:10.1146/annurev-physiol-030212-183708 
5. Beral V, Bull D, Reeves G; Million Women Study Collaborators. Endometrial cancer and hormone-replacement therapy in the Million Women Study. Lancet. 2005;365(9470):1543-1551. doi:10.1016/S01406736(05)66455-0

6. Vinogradova Y, Coupland C, Hippisley-Cox J. Use of hormone replacement therapy and risk of breast cancer: Nested case-control studies using the QResearch and CPRD databases. BMJ. 2020;371:m3873. doi:10.1136/bmj.m3873

7. Cuzick J, Sestak I, Forbes JF, et al. Use of anastrozole for breast cancer prevention (IBIS-II): Long-term results of a randomised controlled trial. Lancet. 2020;395(10218):117-122. Erratum in: Lancet. 2020 Feb 15; 395(10223):496. Erratum in: Lancet. 2021 Feb 27;397(10276):796. doi:10. 1016/S0140-6736(19)32955-1

8. Vogel VG, Costantino JP, Wickerham DL, et al. Effects of tamoxifen vs raloxifene on the risk of developing invasive breast cancer and other disease outcomes: The NSABP study of tamoxifen and raloxifene (STAR) P-2 trial. JAMA. 2006;295(23):2727-2741. Erratum in: JAMA. 2006 Dec 27;296(24):2926. Erratum in: JAMA. 2007 Sep 5;298(9):973. doi:10.1001/jama.295.23.joc60074

9. Sumida C, Lecerf F, Pasqualini JR. Control of progesterone receptors in fetal uterine cells in culture: Effects of estradiol, progestins, antiestrogens, and growth factors. Endocrinology. 1988;122(1):3-11. doi:10.1210/endo-122-1-3

10. National Institute for Health and Care Excellence. Menopause: diagnosis and management: 2019. London, UK: National Institute for Health and Care Exellence; 2019. PMID:31940155

11. Zolfaroli I, Tarín JJ, Cano A. The action of estrogens and progestogens in the young female breast. Eur J Obstet Gynecol Reprod Biol. 2018;230:204-207. doi:10.1016/j.ejogrb.2018.03.057

12. Zolfaroli I, Tarín JJ, Cano A. Hormonal contraceptives and breast cancer: Clinical data. Eur J Obstet Gynecol Reprod Biol. 2018;230:212-216. doi:10.1016/j.ejogrb.2018.03.058

13. Anderson DM, Maraskovsky E, Billingsley $W L$, et al. A homologue of the TNF receptor and its ligand enhance T-cell growth and dendriticcell function. Nature. 1997;390(6656):175-179. doi:10.1038/36593

14. Walsh MC, Choi Y. Biology of the RANKL-RANK-OPG system in immunity, bone, and beyond. Front Immunol. 2014;5:511. doi:10.3389/ fimmu.2014.00511

15. Ahern E, Smyth MJ, Dougall WC, Teng MWL. Roles of the RANKLRANK axis in antitumour immunity - implications for therapy. Nat Rev Clin Oncol. 2018;15(11):676-693. doi:10.1038/s41571-018-0095-y

16. Lacey DL, Timms E, Tan HL, et al. Osteoprotegerin ligand is a cytokine that regulates osteoclast differentiation and activation. Cell. 1998;93(2):165-176. doi:10.1016/s0092-8674(00)81569-x

17. Gonzalez-Suarez E, Branstetter D, Armstrong A, Dinh H, Blumberg H Dougall WC. RANK overexpression in transgenic mice with mouse mammary tumor virus promoter-controlled RANK increases proliferation and impairs alveolar differentiation in the mammary epithelia and disrupts lumen formation in cultured epithelial acini. Mol Cell Biol. 2007;27(4):1442-1454. doi:10.1128/MCB.01298-06

18. González Ricarte M, de Castro Pérez A, Tarín JJ, Cano A. Progestogens and risk of breast cancer: A link between bone and breast? Gynecol Endocrinol. 2016;32:6-8. doi:10.3109/09513590.2015.1078304

19. Gonzalez-Suarez E, Jacob AP, Jones J, et al. RANK ligand mediates progestin-induced mammary epithelial proliferation and carcinogenesis Nature. 2010;468(7320):103-107. doi:10.1038/nature09495

20. Sigl V, Penninger JM. RANKL/RANK - from bone physiology to breast cancer. Cytokine Growth Factor Rev. 2014;25(2):205-214. doi:10.1016/j. cytogfr.2014.01.002

21. Hu H, Wang J, Gupta A, et al. RANKL expression in normal and malignant breast tissue responds to progesterone and is up-regulated during the luteal phase. Breast Cancer Res Treat. 2014;146(3):515-523. doi:10.1007/s10549-014-3049-9
22. González-Suárez E. RANKL inhibition: A promising novel strategy for breast cancer treatment. Clin Transl Oncol. 2011;13(4):222-228. doi:10.1007/s12094-011-0646-5

23. Schramek D, Leibbrandt A, Sigl V, et al. Osteoclast differentiation factor RANKL controls development of progestin-driven mammary cancer. Nature. 2010;468(7320):98-102. doi:10.1038/nature09387

24. Yoldi G, Pellegrini P, Trinidad EM, et al. RANK signaling blockade reduces breast cancer recurrence by inducing tumor cell differentiation. Cancer Res. 2016;76(19):5857-5869. doi:10.1158/0008-5472. CAN-15-2745

25. Park HS, Lee A, Chae BJ, Bae JS, Song BJ, Jung SS. Expression of receptor activator of nuclear factor kappa-B as a poor prognostic marker in breast cancer. J Surg Oncol. 2014;110(7):807-812. doi:10.1002/jso. 23737

26. Widschwendter M, Burnell M, Fraser L, et al. Osteoprotegerin (OPG), the endogenous inhibitor of receptor activator of NF-kappaB ligand (RANKL), is dysregulated in BRCA mutation carriers. EBioMedicine. 2015;2(10):1331-1339. doi:10.1016/j.ebiom.2015.08.037

27. Sigl V, Owusu-Boaitey K, Joshi PA, et al. RANKL/RANK control Brca1 mutation-driven mammary tumors. Cell Res. 2016;26(7):761-774. doi:10. 1038/cr.2016.69

28. Odén L, Akbari M, Zaman T, et al. Plasma osteoprotegerin and breast cancer risk in BRCA1 and BRCA2 mutation carriers. Oncotarget. 2016; 7(52):86687-86694. doi:10.18632/oncotarget.13417

29. Kotsopoulos J, Singer C, Narod SA. Can we prevent BRCA1-associated breast cancer by RANKL inhibition? Breast Cancer Res Treat. 2017; 161(1):11-16. doi:10.1007/s10549-016-4029-z

30. Pfitzner BM, Branstetter D, Loibl S, et al. RANK expression as a prognostic and predictive marker in breast cancer. Breast Cancer Res Treat. 2014;145(2):307-315. doi:10.1007/s10549-014-2955-1.

31. Jones DH, Nakashima T, Sanchez OH, et al. Regulation of cancer cell migration and bone metastasis by RANKL. Nature. 2006;440(7084): 692-696. doi:10.1038/nature04524

32. Gómez R, Castro A, Martínez J, et al. Receptor activator of nuclear factor kappa B (RANK) and clinicopathological variables in endometrial cancer: A study at protein and gene level. Int JMol Sci. 2018;19(7):1848. doi:10.3390/ijms19071848

33. Mørch LS, Kjaer SK, Keiding N, Løkkegaard E, Lidegaard Ø. The influence of hormone therapies on type I and II endometrial cancer: A nationwide cohort study. Int J Cancer. 2016;138(6):1506-1515. doi:10. 1002/ijc. 29878

34. Wang J, Sun X, Zhang H, Wang Y, Li Y. MPA influences tumor cell proliferation, migration, and invasion induced by RANKL through PRB involving the MAPK pathway in endometrial cancer. Oncol Rep. 2015;33(2):799-809. doi:10.3892/or.2014.3651

35. Shi LF, Wu Y, Li CY. Hormone therapy and risk of ovarian cancer in postmenopausal women: a systematic review and meta-analysis. Menopause. 2016;23(4):417-424. doi:10.1097/GME.0000000000000550

36. Wieser V, Sprung S, Tsibulak I, et al. Clinical impact of RANK signalling in ovarian cancer. Cancers (Basel). 2019;11(6):791. doi:10.3390/ cancers 11060791

37. Coleman R, Finkelstein DM, Barrios C, et al. Adjuvant denosumab in early breast cancer (D-CARE): an international, multicentre, randomised, controlled, phase 3 trial. Lancet Oncol. 2020;21(1):60-72. doi:10.1016/S1470-2045(19)30687-4

38. Pantano F, Rossi E, luliani M, et al. Dynamic changes of receptor activator of nuclear factor-KB expression in circulating tumor cells during denosumab predict treatment effectiveness in metastatic breast cancer. Sci Rep. 2020;10(1):1288. doi:10.1038/s41598-020-58339-2 